\title{
A Machine Learning Approach to Predicting Winning Patterns in Track Cycling Omnium
}

\author{
Bahadorreza Ofoghi ${ }^{1,2}$, John Zeleznikow ${ }^{1}$, Clare MacMahon ${ }^{1}$, and Dan Dwyer ${ }^{2}$ \\ 1 Victoria University, Melbourne VIC 3000, Australia \\ 2 Victorian Institute of Sport, Melbourne VIC 8006, Australia
}

\begin{abstract}
This paper presents work on using Machine Learning approaches for predicting performance patterns of medalists in Track Cycling Omnium championships. The omnium is a newly introduced track cycling competition to be included in the London 2012 Olympic Games. It involves six individual events and, therefore, requires strategic planning for riders and coaches to achieve the best overall standing in terms of the ranking, speed, and time in each individual component. We carried out unsupervised, supervised, and statistical analyses on the men's and women's historical competition data in the World Championships since 2008 to find winning patterns for each gender in terms of the ranking of riders in each individual event. Our results demonstrate that both sprint and endurance capacities are required for both men and women to win a medal in the omnium. Sprint ability is shown to have slightly more influence in deciding the medalists of the omnium competitions.
\end{abstract}

\section{Introduction}

The Omnium is a track cycling competition that has newly been introduced as one of the events in the Olympic Games by the International Cycling Union (UCI) ${ }^{1}$. The main motivation behind inclusion of the omnium in the Olympic Games is to create a better balance between the number of cycling competitions for men and women in the Olympics. In the Beijing 2008 Olympics, there were seven cycling competitions for men (team sprint, sprint, keirin, 4000-meter team pursuit, 4000-meter individual pursuit, 50-kilometer Madison, and 40-kilometer points race) whereas only three events were held for women (sprint, 3000-meter individual pursuit, 25-kilometer points race). According to the agreement made between UCI and the International Olympic Committee (IOC), in the London 2012 Olympics, the cycling competitions will involve five events per gender including the sprint, keirin, team sprint, team pursuit, and omnium.

The omnium was first included in men's cycling competitions at the UCI Track Cycling Championships in Spain in 2007. Primarily, the omnium included five individual events:

- Flying time trial, where cyclists compete in a short flying lap that has traditionally been used for qualification in other cycling events (e.g. individual pursuit).

$\overline{1}$ http://www.uci.ch

M. Bramer (Ed.): IFIP AI 2010, IFIP AICT 331, pp. 6776, 2010.

(C) IFIP International Federation for Information Processing 2010 
- Scratch race, in which all contestants start from a start point at the same time and need to complete a certain number of laps. If a rider gains an entire lap ahead of the other riders, she/he will have an advantage over the riders who have completed fewer laps.

- Individual pursuit, where two riders start a race from opposite sides of the track on the pursuit line at the bottom of the track. The riders start at the same time and both must complete the race distance to record a time for the ranking.

- Points race, which is a mass start event involving a large number of riders on the track at the same time. This is a race over a long distance (about 120 to 160 laps). Every 10 laps, a sprint is held and the top four finishing riders are awarded 5, 3, 2, and 1 points respectively. Any rider who can complete a lap is also awarded 20 points for each lap. In the end, the rider with the most points is the winner of the event.

- (Kilometer) Time trial, where riders compete against the clock to secure the fastest time. Riders are sent out individually in set time intervals. This event includes cycling in moderately longer distances than the flying time trial event.

Each rider in the omnium scores according to their rank in the individual events and the rider with the lowest score wins the competition.

In December 2009, UCI announced new changes to the omnium competition that will take place for the first time in the Olympic Games in London in 2012. The new changes were introduced to bring the balance back towards the endurance riders. According to UCI, the new omnium competition will now involve six events with the following format:

- Flying time trial: 250 meters for men and women

- Scratch race: 15 kilometers for men and 10 kilometers for women

- Individual pursuit: 4 kilometers for men and 3 kilometers for women

- Points race: 30 kilometers for men and 20 kilometers for women

- (Kilometer) Time trial: 1 kilometer for men and 500 meters for women

- Elimination race: 24 riders will compete in a race where every two laps, the last rider over the finish line will be eliminated until only a single rider remains and is decided as the winner.

The elimination race has never been part of the omnium, which creates an unknown factor in regards to predicting the overall winner. The omnium competition with its new format will be held over two days. The sequence of the events in each day has yet to be announced by UCI.

\section{Related Work}

Because of the nature of multiple-component competitions comprised individual events, it is difficult to determine what strategies need to be employed by coaches and athletes in order to maximize the chances of winning the whole competition. 
In similar sports to the omnium such as the Decathlon (with 10 individual track and field events), overall success can be attributed to different performances for each individual component. Kenny et al. 11 argue that excellence in any individual event at the decathlon competitions may be achieved at the expense of finishing the whole competition with a poorer overall standing. Cox and Dunn [2], however, used statistical analysis on the data collected at the meetings of the International Amateur Athletic Federation (1991 - 1999) and concluded that the decathlon favors athletes who are proficient in the field events.

Zwols and Sierksma [3] studied training data from the decathlon. They made use of mathematical optimization methods to find the best combinations of training times/sessions for performing well in different types of championships/ competitions. As they also make the point, it is, however, not straightforward to relate training performances to competition performances as there are other factors (such as mental/psychological preparedness) that can potentially interfere with performance outcomes.

Much of the previous work in the domain of sports data analysis is based on the statistical evidence. Although statistical analysis is a good approach to make better sense of sports data, it should not be taken as the only source of information that can be extracted from the data. Data mining and machine learning-based approaches have also been used widely in this domain. Examples include modeling and predicting competitive performances in swimming using Neural Networks 4, using Self Organizing Maps for classifying coordination patterns of participants in different types of basketball shots [5], cluster analysis of elite swimmers' race patterns [6], predicting player injuries in soccer using data mining 7], and knowledge discovery techniques implemented in Advanced Scout software used for finding series of consecutive events that may lead to scoring in NBA basketball games [8].

In this paper, we also employ machine learning-based approaches and try to confirm our results on the strategic planning for winning a medal in the omnium competitions by conducting a further complementary statistical analysis.

\section{$3 \quad$ Research Problems}

In terms of the omnium, especially with its new format, there is little to no previous research to the best of our knowledge. With this event to be included in the Olympics for the first time, it is, however, very useful for both coaches and athletes to gain a better understanding of the strategies to be employed and assist them towards winning the competition.

In particular, for both male and female cyclists, we would like to understand:

1. What overall ranking in each individual event is required to win a medal in the omnium?

2. What type of rider may have a greater advantage with regards to winning a medal in the omnium? Those with sprint or endurance capacity?

3. What individual events have more influence on the final standing in the omnium? 
We used a number of unsupervised and supervised learning mechanisms as well as statistical analysis methods to answer these questions and assist coaches and riders with a better strategic plan for winning a medal in the omnium. We did not attempt at developing new artificial intelligence methods for this; instead, tried to see how existing methods can be used/adjusted in this context.

\section{Data Collection}

Since omnium competitions were only introduced to the World Championships after 2006, there is only presently a limited dataset available. The data that we have collected consist of the competition results for the following events:

- The 2009 Track Cycling World Championships (women, men)

- The 2009 Australian Track Championships (women, men)

- The 2009 Juniors Track Cycling World Championships (women, men)

- The 2008 Juniors Track Cycling World Championships (men)

The dataset includes 96 data records for men and 75 data records for women. Since there is inconsistency in the dataset in terms of the class of the riders (i.e., masters and juniors), it is not viable to conduct a study on non-relative attributes such as time, speed, and score for each individual event. Therefore, we only considered the ranking/placing of the riders in each individual event.

On the other hand, since the elimination race is a totally new individual event in the omnium, previous data/results do not involve any information regarding this event in the context of the omnium.

Therefore, our dataset only includes the ranking of the cyclists at the individual events excluding the elimination race and the overall final standing of the riders in omnium competitions.

\section{Empirical Analysis}

In order to answer the questions raised in section 3. we first pre-processed the dataset. Pre-processing included two steps:

1. Converting the data to the format that can be dealt with by the machine learning package that we use (the weka machine learning packag£). This mainly consists of replacing missing values with a character (the question mark) and inserting the header information at the beginning of the data file.

2. Categorizing/generalizing the final standings in the omnium in to pre-defined classes:

- 1: Any medal winners including the final standings $1^{\text {st }}, 2^{\text {nd }}$, and $3^{\text {rd }}$,

- 2: Non-medal winners ranked between 4 and 10 inclusive, and

- 3: Non-medal winners ranked above 10.

After pre-processing was completed, we carried out different experiments to find answers to the research problems already mentioned.

\footnotetext{
${ }^{2}$ http://www.cs.waikato.ac.nz/ml/weka/
} 


\subsection{Experiment 1 - Unsupervised Learning of Rankings}

The unsupervised learning experiment included a cluster analysis of the data records collected for the riders in different competitions. We used the $K$-Means clustering algorithm implemented in weka and set the number of clusters to 3 . The three clusters were selected on the basis of the three categories of the final standings. We ran the K-Means algorithm on the men's and women's data separately. Table 1 and Table 2 summarize the results for each gender.

Table 1. Unsupervised (clustering) learning of rankings for each individual event on the men's competition results. Numbers in parentheses represent the number of instances/records in each cluster. Within cluster sum of squared errors $=16.1598$.

\begin{tabular}{|c|c|c|c|c|}
\hline Attribute & Full data (96) & Cluster 1 (21) & Cluster $2(15)$ & Cluster 3 (60) \\
\hline$\overline{\text { PR_rank }}$ & 7.8676 & 11.0476 & 3.8667 & 7.7549 \\
\hline TT_rank & 8.6774 & 11.4808 & 5.5828 & 8.4699 \\
\hline IP_rank & 8.0000 & 13.0000 & 3.1333 & 7.4667 \\
\hline SR_rank & 9.0588 & 11.5266 & 7.2902 & 8.6373 \\
\hline FTT_rank & 8.2877 & 13.0137 & 3.8667 & 7.7388 \\
\hline final_standing & 2.0000 & 3.0000 & 1.0000 & 2.0000 \\
\hline
\end{tabular}

$\mathrm{PR}=$ Points Race, $\mathrm{TT}=$ Time Trial, $\mathrm{IP}=$ Individual Pursuit, $\mathrm{SR}=$ Scratch Race, $\mathrm{FTT}=$ Flying Time Trial.

The column "Full data" represents the average values (means) of each attribute over the full set of data, whereas the other columns show the average values over the instances in each cluster.

From the results in both Table 1 and Table 2, it is not surprising that the average final standing under the column "Full data" is equal to 2 since most of the data records have the final standing of category 2 (finishing in position 4 to 10). It can also be seen that the clustering algorithm has correctly detected three clusters for men's and women's data where the final standings cover the three categories of final standings that we set. This is an important observation since it shows that there is a pattern of the rankings in individual events that correspond to each final standing category. In other words, the clustering algorithm has been able to bring together data points with similar final standings in certain groups.

For men, the results in Table 1 suggest that in order for a rider to win a medal (final_standing $=1$, Cluster 2), it is required to achieve the rankings of approximately $4,5,3,7$, and 4 in the points race, time trial, individual pursuit, scratch race, and flying time trial events respectively. In general, the flying time trial and individual pursuit components require strong sprint capacity, whereas the scratch race and time trial events are dealt with much better by riders with endurance capacity. The points race event requires a mixture of sprint and endurance abilities. Therefore, our results suggest that in order for a male rider to win a medal in the omnium it is required that he has a combination of sprint and endurance power without either being an outstanding sprinter or having excellent endurance ability. In fact, the endurance capacity can be neglected to 
Table 2. Unsupervised (clustering) learning of rankings for each individual event on the women's competition results. Numbers in parentheses represent the number of instances/records in each cluster. Within cluster sum of squared errors $=11.4309$.

\begin{tabular}{|c|c|c|c|c|}
\hline Attribute & Full data (75) & Cluster $1(14)$ & Cluster $2(46)$ & Cluster 3 (12) \\
\hline PR_rank & 7.5577 & 10.8255 & 7.5973 & 3.5833 \\
\hline TT_rank & 8.0000 & 13.2857 & 6.9184 & 6.2500 \\
\hline IP_rank & 7.9123 & 13.0714 & 7.4168 & 3.9167 \\
\hline SR_rank & 8.7576 & 11.1082 & 8.6481 & 6.4621 \\
\hline FTT_rank & 8.0847 & 13.8571 & 7.6603 & 3.0833 \\
\hline final_standing & 2.0000 & 3.0000 & 2.0000 & 1.0000 \\
\hline
\end{tabular}

some degree as the results show that the ranking of the rider in the scratch race can be as low as about 7 while winning a medal.

For women, the results in Table 2 show, however, in order to win a medal, more sprinting capacity is required with only moderate endurance power. This is a result of the average rankings under Cluster 3 with final_standing $=1$. A ranking between 3 and 4 in the points race, individual pursuit, and flying time trial events in addition to final rankings of about 6 in the time trial and scratch race components can potentially lead to winning a medal for a female cyclist.

\subsection{Experiment 2 - Supervised Learning of Rankings}

To see how accurate the predictions in Experiment 1 are, for both men and women, we conducted a supervised learning experiment. The supervised learning experiment consists of a classification task that classifies each instance/data record into one of the predefined categories of the final standing explained in section 5. For this, we used the Naive Bayes classifier implemented in weka and conducted a 10-fold cross validation process for testing the classification accuracies. In this experiment, we did not consider any inter-dependence between the different attributes (i.e. rankings in each individual event). Table 3 and Table 4 show the results of the classification task for men and Table 5 and Table 6] summarize the results of the same analyses for women.

For men, the results in Table 3 demonstrate that for instance if a rider finishes ranked $4,3,3,6$, and 4 in the points race, time trial, individual pursuit, scratch race, and flying time trial events respectively, then there is a chance of approximately $84 \%$ that he/she can win a medal in the omnium competition. It is promising to see that the trend of the results of the supervised learning analysis matches that of the results of the unsupervised learning analysis in terms of the average ranking in each individual event. To illustrate this, one might compare the average rankings under Cluster 2 in Table 1 with the mean values under final_standing $=1$ in Table 3 .

The supervised analysis on the male athlete data again shows that both sprint and endurance capacities are required to win a medal with a slightly lesser endurance power than sprint ability. According to our analysis, finishing ranked 
Table 3. Supervised (classification) learning of rankings for each individual event on the men's competition results. Numbers in parentheses represent the percentage of instances/records in each class.

\begin{tabular}{|c|c|c|c|c|}
\hline \multicolumn{2}{|l|}{ Attribute } & \multirow{2}{*}{$\frac{\text { final_st. }=1(0.22)}{3.8667}$} & \multirow{2}{*}{$\begin{array}{l}\text { final_st. }=2(0.47) \\
7.6563\end{array}$} & \multirow{2}{*}{$\frac{\text { final_st. }=3(0.31)}{11.0476}$} \\
\hline$\overline{P R \_r a n k}$ & mean & & & \\
\hline & std. dev. & 2.1868 & 4.5662 & 3.9094 \\
\hline \multirow[t]{2}{*}{ TT_rank } & mean & 2.8750 & 7.7778 & 13.5833 \\
\hline & std. dev. & 1.6154 & 2.9355 & 1.8465 \\
\hline \multirow[t]{2}{*}{ IP_rank } & mean & 3.1333 & 6.7500 & 13.0000 \\
\hline & std. dev. & 2.0613 & 3.0822 & 3.2514 \\
\hline \multirow[t]{2}{*}{ SR_rank } & mean & 6.1111 & 7.4286 & 11.6500 \\
\hline & std. dev. & 4.8177 & 4.0891 & 4.2694 \\
\hline \multirow[t]{2}{*}{ FTT_rank } & mean & 3.8667 & 7.5625 & 13.2500 \\
\hline & std. dev. & 2.4459 & 3.4635 & 3.6038 \\
\hline \multicolumn{3}{|c|}{ Correctly classified instances } & 58 & $84.0580 \%$ \\
\hline \multicolumn{3}{|c|}{ Incorrectly classified instances } & 11 & $15.9420 \%$ \\
\hline
\end{tabular}

Table 4. Classification measures for supervised learning of rankings for each individual event on the men's competition results

\begin{tabular}{lcccc}
\hline Class & Precision & Recall & F-measure & ROC area \\
\hline final-standing=1 & 0.833 & 0.667 & 0.741 & 0.927 \\
final-standing=2 & 0.789 & 0.909 & 0.845 & 0.886 \\
final-standing=3 & 0.947 & 0.857 & 0.900 & 0.959 \\
Weighted average & 0.847 & 0.841 & 0.839 & 0.917 \\
\hline
\end{tabular}

about 6 in the scratch race is sufficient for winning the omnium given the rankings in the other events mentioned in the previous paragraph.

The results in Table 4 show the accuracy level of these arguments in more detail. The most accurate predictions (with an accuracy of approximately 95\%) for where a competitor will place in the omnium, based on performance in the separate events, can be made for those who finish outside the top ten (final_standing $=3)$. For the medal winners, the accuracy is slightly lower $(\sim 84 \%)$ and the lowest accuracy corresponds to the non-medal winners who may finish the competition with a final standing between 4 and 10 inclusive $(\sim 79 \%)$.

Overall, the average precision, recall, F-measure, and ROC area are all above $83 \%$ and this is a promising result emphasizing the correctness of concluding remarks drawn from the unsupervised analysis for men (see section [5.1).

For women, the results summarized in Table 5 suggest similar conclusions to those drawn from the men's dataset. Once again, the only major difference is the higher average rank (5.00) required for the time trial component compared to that for men (2.8750). As with the unsupervised analysis, this suggests that, for women, there is more bias towards sprinting capacity to win the omnium. 
Table 5. Supervised (classification) learning of rankings for each individual event on the women's competition results. Numbers in parentheses represent the percentage of instances/records in each class.

\begin{tabular}{|c|c|c|c|c|}
\hline \multicolumn{2}{|l|}{ Attribute } & \multirow{2}{*}{$\begin{array}{l}\text { final_st. }=1(0.24) \\
3.5833\end{array}$} & \multirow{2}{*}{$\begin{array}{l}\text { final_st. }=2(0.49) \\
7.2308\end{array}$} & \multirow{2}{*}{$\begin{array}{l}\text { final_st. }=3(0.27) \\
11.0769\end{array}$} \\
\hline$\overline{P R \_r a n k}$ & mean & & & \\
\hline & std. dev. & 3.0675 & 3.4453 & 3.9703 \\
\hline \multirow[t]{2}{*}{ TT_rank } & mean & 5.0000 & 7.3000 & 13.6923 \\
\hline & std. dev. & 2.5071 & 3.4799 & 2.0147 \\
\hline \multirow[t]{2}{*}{ IP_rank } & mean & 3.9167 & 6.8846 & 13.0714 \\
\hline & std. dev. & 2.5317 & 3.5008 & 3.3480 \\
\hline \multirow[t]{2}{*}{ SR_rank } & mean & 4.1667 & 8.6429 & 11.5000 \\
\hline & std. dev. & 2.9107 & 4.2191 & 4.2720 \\
\hline \multirow[t]{2}{*}{ FTT_rank } & mean & 3.0833 & 7.7308 & 13.8571 \\
\hline & std. dev. & 1.6562 & 3.2999 & 2.3561 \\
\hline \multicolumn{3}{|c|}{ Correctly classified instances } & 45 & $86.5385 \%$ \\
\hline \multicolumn{3}{|c|}{ Incorrectly classified instances } & 7 & $13.4615 \%$ \\
\hline
\end{tabular}

Table 6. Classification measures for supervised learning of rankings for each individual event on the women's competition results

\begin{tabular}{lcccc}
\hline Class & Precision & Recall & F-measure ROC area \\
\hline final-standing=1 & 0.818 & 0.750 & 0.783 & 0.896 \\
final-standing=2 & 0.852 & 0.885 & 0.868 & 0.857 \\
final-standing=3 & 0.929 & 0.929 & 0.929 & 0.980 \\
Weighted average & 0.865 & 0.865 & 0.865 & 0.899 \\
\hline
\end{tabular}

The overall accuracy of the classification task is about $86 \%$, slightly higher than that for men. The detailed classification measures shown in Table 6 demonstrate a slightly higher overall precision, recall, F-measure, and ROC area for women.

\subsection{Experiment 3 - Statistical Analysis of Individual Events}

In order to understand what individual events have more influence on the final standing in the omnium, we carried out a statistical analysis on the relationship between the values of each attribute (rankings) in our dataset and the values of the attribute that represents the final standing of the riders. For this, we used the Correlation Coefficient measure $r$. We back tracked in the pre-processing step (generalizing the final standings to classes 1, 2, and 3) and used the raw final standing values ranging from 1 to the number of riders in each competition.

The results of the statistical analysis for men and women are shown in Figure 1 and Figure 2. For both genders, the highest correlations are between the individual pursuit rank and flying time trial rank and the final standing. Especially in the case of women, this is very much consistent with the results of the unsupervised and supervised learning processes where sprinting capability has 


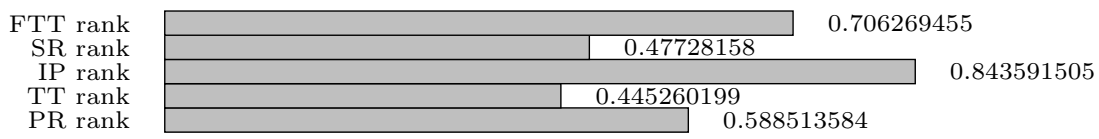

Fig. 1. Correlation coefficient measures between the rankings in each individual event and the final standings of male riders

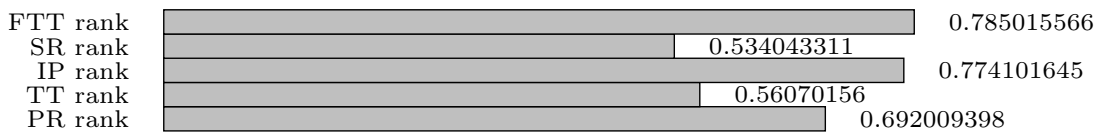

Fig. 2. Correlation coefficient measures between the rankings in each individual event and the final standings of female riders

been shown to be more important for a female rider to win omnium competitions. For men, this is slightly less consistent, however.

For women, the lowest correlation is between the scratch race rank and the final standing, whereas for men, the least correlation corresponds to the time trial rank and the final standing. In both cases, this emphasizes that endurance power, although required, can play a less important role in winning the omnium.

It is important to note that the results of our statistical analysis using the correlation coefficient $r$, however, do not show the causation relationship but only a correlation relationship that can be used in combination with the results of other experiments to provide strength to the interpretations of the results.

\section{Conclusion and Future Work}

We studied the newly introduced Olympic track cycling competition, the omnium, to understand what performance, in terms of ranking, in each individual event of this competition is required to win a medal, what type of riders may have the greatest chance of winning a medal (riders with sprint or endurance capacity), and what individual events may have more influence on the final standing in the omnium. For this, we used machine learning and statistical approaches to analyze the competition data available to us so far.

The results of our unsupervised clustering, supervised classification, and statistical correlation coefficient analysis suggest that both sprint and endurance capacities are required for both men and women to win a medal in the omnium. However, especially in the case of women, there can be less emphasis on endurance power and more on sprint ability. This is a result of our machine learning-based analyses and is supported by our statistical analysis that shows high correlations between the rankings of the riders in the sprint-power-requiring individual events (i.e., flying time trial and individual pursuit) and the final standings in the omnium. These results are based on a form of the omnium that 
is about to change (to a six-event competition); therefore, our contributions may change when we repeat the analyses on competition results in the future.

Because of the inconsistent nature of our database in terms of speed, time, and scores of individual events, we are trying to collect more related data and repeat our experiments with a larger dataset that includes a greater number of data records for junior and senior men and women cyclists. This will give us the opportunity to carry out analyses on other more abstract attributes time, speed, and scores achieved by riders.

Other current work is analyzing the inter-dependence between different attributes (features of individual events) to construct a probabilistic model to tell us that, given certain ranks, speeds, times, and/or scores in certain individual events, what may be achieved by riders in the other individual events and potentially in the omnium competition.

\section{References}

1. Kenny, I.C., Sprevak, D., Sharp, C., Boreham, C.: Determinants of success in the Olympic decathlon: Some statitistical evidence. Journal of Quantitative Analysis in Sports 1 (2005)

2. Cox, T.F., Dunn, R.T.: An analysis of decathlon data. Journal of the Royal Statistical Society 51, 179-187 (2002)

3. Zwols, Y., Sierksma, G.: Training optimization for the decathlon. Journal of Operations Research 57, 812-822 (2009)

4. Edelmann-nusser, J., Hohmann, A., Henneberg, B.: Modeling and prediction of competitive performance in swimming upon neural networks. European Journal of Sport Science 2, 1-10 (2002)

5. Choi, H.: Cluster analysis of Korean elite athletes' fitness using the self organised map. In: Proceedings of International Association for Computer Science in Sport, Australian Institute of Sport, Canberra, Australia (2009)

6. Chen, I., Homma, H., Jin, C., Hong Yan, H.: Identification of elite swimmers' race patterns using cluster analysis. International Journal of Sports Science and Coaching 3, 293-303 (2007)

7. Flinders, K.: Football injuries are rocket science. vnunet.com (2002), http://www.vnunet.com/vnunet/news/2120386/football-injuriesrocketscience

8. Bhandari, I., Colet, E., Parker, J., Pines, Z., Pratap, R., Ramanujam, K.: Advanced Scout: Data mining and knowledge discovery in NBA data. Data Mining and Knowledge Discovery 1, 121-125 (2004) 